\title{
A retrospective cohort study of clinical outcomes for intravitreal crystalline retained lens fragments after age-related cataract surgery: a comparison of same-day versus delayed vitrectomy
}

\author{
This article was published in the following Dove Press journal: \\ Clinical Ophthalmology \\ 17 July 2012 \\ Number of times this article has been viewed
}

\section{Elizabeth A Vanner' \\ Michael W Stewart ${ }^{2}$ \\ Thomas J Liesegang ${ }^{2}$ \\ Rick E Bendel ${ }^{2}$ \\ James P Bolling ${ }^{2}$ \\ Saiyid A Hasan²}

'Departments of Preventive

Medicine and Health Care Policy and Management, Stony Brook University, Stony Brook, NY; ${ }^{2}$ Department of Ophthalmology, Mayo Clinic College of Medicine, Jacksonville,

FL, USA
Correspondence: Michael W Stewart Department of Ophthalmology, Mayo Clinic College of Medicine, 4500 San Pablo Road, Jacksonville, FL 32224, USA

Tel +l 9049532232

Fax +l 9049537040

Email stewart.michael@mayo.edu
Background: This analysis compared outcomes for same-day (under a no-move, no-wait policy) versus delayed vitrectomy for intravitreal crystalline retained lens fragments after surgery for age-related cataract.

Methods: This was a retrospective, nonrandomized treatment comparison cohort study with a consecutive series of 35 eyes ( 23 same-day, 12 delayed) receiving both cataract surgery and vitrectomy at the Mayo Clinic Florida between 1999 and 2010. Outcome measures included visual acuity (VA), glaucoma progression, visual utility, and complications. Several techniques (bootstrapping, robust confidence intervals, jackknifing, and a homogeneous sample) were used to reduce selection bias and increase confidence in our small sample's results.

Results: No significant baseline treatment group differences. Mean previtrectomy delay (12 eyes) was 40.9 days (median 29.5, range 1-166). Mean postvitrectomy follow-up (35 eyes) was 47.5 months (median 40.5, range 3.1-123.5). Same-day patients had significantly better final VA (adjusted for age $[t=-2.14, P=0.040]$ and precataract surgery VA $[t=-2.98, P=0.006]$ ); a higher rate of good final VA ( $\geq 20 / 40), 78.3 \%(18 / 23)$ versus $58.3 \%(7 / 12)$; a lower rate of bad final VA ( $\leq 20 / 200), 4.3 \%(1 / 23)$ versus $25.0 \%$ (3/12); and fewer final retinal conditions, $4.3 \%(1 / 23)$ versus $50.0 \%(6 / 12)$. Same-day patients also had marginally significant better mean final VA in the operated eye ( $20 / 40$ versus $20 / 90, Z=1.51, P=0.130)$ despite poorer initial VA (20/98 versus $20 / 75)$ and higher age ( $3+$ years), better final visual utility, and longer survival times for better VA. Among patients with preexisting glaucoma, same-day patients experienced significantly less differential (operated versus nonoperated eye) glaucoma progression.

Conclusion: Results favored same-day patients, who experienced better final VA and visual utility, less differential glaucoma progression, and fewer complications. Results need confirmation with larger samples.

Keywords: intraoperative complications, retained lens fragments, visual acuity, glaucoma, evaluation studies, visual utility, statistics as topic, small nonrandomized sample analysis

\section{Introduction}

Phacoemulsification cataract surgery occasionally results in fragments or the entire crystalline lens dislocating into the vitreous. There is evidence that the clinical course for patients with retained lens fragments (RLF) begins the instant the fragments enter the vitreous and is affected by the cataract surgeon's decisions and actions. ${ }^{1}$ Lack of immediate availability of an experienced vitreoretinal surgeon and the necessary equipment usually precludes a same-day vitrectomy, which many suggest might be the optimal time for RLF removal. ${ }^{2-6}$ 
Studies comparing same-day and delayed vitrectomy show mixed results. Several authors reported better visual acuity $(\mathrm{VA})^{7-9}$ and lower rates of complications - retinal detachment (RD), ${ }^{7-10}$ corneal edema, ${ }^{10,11}$ glaucoma/elevated intraocular pressure (IOP), ${ }^{7-12}$ cystoid macular edema (CME), ${ }^{7,8,10}$ and intraocular inflammation/infection ${ }^{7,10,11}$ - among same-day patients. Lower rates of elevated IOP and/or CME may be related to less intraocular inflammation/infection. ${ }^{8,10}$ Others reported no significant differences in $\mathrm{VA},{ }^{13} \mathrm{RD},{ }^{12}$ and $\mathrm{IOP}^{13}$ between same-day and delayed vitrectomy patients.

This study compared outcomes of patients with RLF who received a same-day vitrectomy, under the Mayo Clinic Florida (MCF) "no move, no wait" policy, versus a delayed vitrectomy any day after cataract surgery. The MCF policy is that a same-day vitrectomy can be performed only if a vitreoretinal surgeon begins the procedure within 15 minutes of cataract surgery and the patient is not moved from the original operating room. Outcomes included VA, visual utility, new glaucoma cases, differential progression of preexisting glaucoma, and ocular complications. This study received institutional review board approval and an informed consent waiver from the MCF Institutional Review Board.

\section{Methods}

\section{Patients, study design, and setting}

This was a retrospective cohort study with a consecutive series of 34 patients ( 35 eyes) who had received both cataract surgery and vitrectomy at MCF (1999-2010). Inclusion criteria were scheduled phacoemulsification cataract surgery for an age-related cataract and intravitreal crystalline RLF managed surgically with a standard three-port pars plana vitrectomy. Twelve eyes (exposed to RLF for 1+ days) received a delayed vitrectomy (1999-2004) with mean delay of 40.9 days (median 29.5, range 1-166). Indications for vitrectomy in this delayed group included elevated IOP, excessive inflammation, CME, and/or decreased VA. Same-day vitrectomy (1999-2010) was successfully performed on 22 eyes and attempted on the remaining eye, but not completed due to an equipment problem. This patient's data were analyzed in the same-day group, according to intention-to-treat, ${ }^{14}$ yielding an unexposed group with 23 eyes. All patients returned for follow-up visits (mean postvitrectomy follow up 47.5 months [median 40.5, range 3.1-123.5]) as per the standard of care, and data was abstracted from patients' medical records.

\section{Variables}

All variables pertain to the operated eye unless otherwise noted. Snellen VA, measured during all visits, was converted to logarithm of the minimum angle of resolution ( $\log M A R)$ for statistical analysis. ${ }^{15}$ Total elapsed time was from cataract surgery to final visit. The following were considered final retinal conditions: CME, RD, background diabetic retinopathy with focal laser photocoagulation, macular scar, and/or drusen; and we included the following as final corneal conditions: superficial scar, mild edema, and/or mild haze. We defined good VA as $\geq 20 / 40$, bad VA as $\leq 20 / 200$, and ocular inflammation as the presence of cells in the anterior chamber.

New glaucoma cases included patients requiring longterm pressure-lowering drops (in the operated eye) after vitrectomy who did not require this before cataract surgery. Glaucoma progression was defined as the exacerbation of visual field abnormalities, calculated by subtracting precataract surgery mean deviation (MD) from final MD and precataract surgery pattern-specific deviation (PSD) from final PSD. Differential glaucoma progression was calculated (separately for MD and PSD) by subtracting glaucoma progression in the nonoperated (fellow) eye from glaucoma progression in the operated eye.

Two scales were used for final visual utility: visual utility - better eye (based mainly on VA in the better eye) ${ }^{16}$ and visual utility - both eyes (adapted to use only VA data but from both eyes ${ }^{17}$ because RLF typically occur only in a single eye, often the one with worse VA). Because it is patient-specific (not eye-specific), the visual utility analyses contained only 22 same-day patients because one patient received a same-day vitrectomy in both eyes.

\section{Data analyses}

All analyses compared results for patients' operated eyes by treatment group (same-day versus delayed) and were preformed using Stata/IC version 11.1 (StataCorp, College Station, TX, USA). When doing the analyses, exact tests (which do not rely on large-sample assumptions of asymptotic normality) were used whenever possible. ${ }^{18}$ In addition, model adjustment for potential confounding variables was limited because, in a small dataset, including too many variables in a model might result in overfitting. ${ }^{19}$ However, it was important to adjust these analyses for potential confounding variables to reduce the effects of selection bias, which is inherent when comparing nonrandomized samples. ${ }^{14,20}$ Potential confounding variables included patient age (at final visit), VA precataract surgery (the best proxy available for ocular health and vision before the RLF), and total elapsed time. See the appendix, which describes additional steps taken to reduce effects of selection bias and increase confidence in our small sample results. 
Continuous variables were assessed for normal distributions. Three types of models were built: analysis of covariance (ANCOVA) for final VA and final visual utility - better eye, ordinal logistic regression for final visual utility - both eyes, and exact Cox proportional-hazards regression for time from vitrectomy until VA decreased to and remained $<20 / 40$ and $\leq 20 / 200$. An independent-samples $t$-test compared differential MD glaucoma progression, a Mann-Whitney test compared differential PSD glaucoma progression, and Fisher's exact tests compared complication rates.

Results were considered statistically significant with $P$-values $\leq 0.05 .^{21,22}$ When appropriate, marginally significant differences ( $P \leq 0.15$ ) were noted because small samples are often underpowered to detect significant differences, even when treatment effects are real. ${ }^{14}$ Noting marginally significant results is as appropriate as a multiplicity of post hoc power analyses, and probably more informative, since it is specifically the marginally significant results which should be included in the hypotheses of larger studies to verify the small sample's results. Discussing marginally significant results attempts to reduce the effect of type II errors (the failure of a statistical test to detect actual treatment group differences, when they exist), which are a problem associated with smallsample studies. ${ }^{14}$

\section{Results}

During the study period (1999-2010), 7565 patients had scheduled age-related cataract surgery at MCF, so these 35 cases indicate an RLF incidence rate of $0.46 \%$. All 35 RLF cases were included in this study. Table 1 contains descriptive statistics and treatment group comparisons. There were no significant (or marginally significant) baseline group differences in any precataract surgery variable. There was a significant difference in the volume of RLF (same-day patients having larger amounts, $P<0.001$ ) and a marginally significant difference in intraocular lens (IOL) type (more same-day patients had anterior-chamber IOLs, $P=0.084)$. For postvitrectomy variables, there were significant differences in both MD and PSD differential glaucoma progression (discussed below) and final retinal conditions ( same-day $=4.3 \%$, delayed $=50.0 \% ; P=0.003$ ). There were also marginally significant differences in unadjusted final VA in the operated eye (same-day $=0.30$, delayed $=0.65$; $\mathrm{Z}=1.51, P=0.130$ ), age at final visit (same-day $=84.43$, delayed $=81.37 ; \mathrm{Z}=-1.53, P=0.126$ ), bad final VA (sameday $=4.3 \%[1 / 23]$, delayed $=25.0 \%[3 / 12] ; P=0.106)$, and final visual utility - both eyes (same-day $=0.94$, delayed $=0.91 ; Z=1.54, P=0.124$ ). For all postvitrectomy clinical variables with significant or marginally significant differences, same-day patients had better results. Figure 1 compares precataract surgery and final VA (operated eye) and differential glaucoma progression (operated versus nonoperated eye) by treatment group.

Table 2 displays descriptive statistics and normal distribution test results. For three dependent variables, final logMAR VA in the operated eye, visual utility - both eyes, and differential PSD glaucoma progression, a normal distribution could not be assumed. Analogous ANCOVA models, built with Snellen (normally distributed) and $\log$ MAR final VA, produced similar results. We report only the logMAR final VA models' results, because it is a continuous variable, ${ }^{15}$ as required by ANCOVA. Attempts to transform the other two variables to normal distributions were unsuccessful.

The models' results are summarized in Table 3. ANCOVA (Table 4) indicated that same-day patients had significantly better adjusted final VA (models 1A and 1B) (age-adjusted difference $=-0.42,95 \%$ confidence interval $[\mathrm{CI}]=-0.83$, $-0.02, t=-2.14, P=0.040$ and precataract surgery VAadjusted difference $=-0.45,95 \% \mathrm{CI}=-0.75,-0.14$, $t=-2.98, P=0.006)$ and marginally significant higher adjusted visual utility - better eye (models $2 \mathrm{~A}$ and $2 \mathrm{~B}$ ), approximately the difference between $20 / 25$ and 20/30 (age-adjusted difference $=0.04,95 \% \mathrm{CI}=-0.01,0.10$, $t=1.62, P=0.116$ and precataract surgery VA-adjusted difference $=0.04,95 \% \mathrm{CI}=-0.01,0.09, t=1.48, P=0.149$ ). Table 3 shows the results of using these ANCOVA models to estimate the patients' mean final VA and visual utility - better eye if the patients had been in the other treatment group.

Ordinal logistic regression (Table 4, models 3A and 3B) indicated that same-day patients had significantly higher odds of better adjusted visual utility - both eyes (age-adjusted odds ratio $=7.38,95 \% \mathrm{CI}=1.12,48.78, \mathrm{Z}=2.07, P=0.038$ and precataract surgery VA-adjusted odds ratio $=7.26,95 \%$ $\mathrm{CI}=1.06,49.66, \mathrm{Z}=2.02, P=0.043)$. Cox regressions (Table 5) indicated that same-day patients had lower hazards for VA decreases for both time-to-VA $<20 / 40$ (models $4 \mathrm{~A}$ and 4B) (age-adjusted hazard ratio $=0.34,95 \% \mathrm{CI}=0.01$, $1.19, \mathrm{Z}=-1.69, P=0.092$, marginally significant, and precataract surgery VA-adjusted hazard ratio $=0.17,95 \%$ $\mathrm{CI}=0.03,0.87, \mathrm{Z}=-2.13, P=0.033$, significant) and timeto-VA $\leq 20 / 200$ (models 5A and 5B) (age-adjusted hazard ratio $=0.16,95 \% \mathrm{CI}=0.02,1.67, \mathrm{Z}=-1.53, P=0.126$ and precataract surgery VA-adjusted hazard ratio $=0.07$, $95 \% \mathrm{CI}=0.00,1.06, \mathrm{Z}=-1.92, P=0.055$, both marginally significant). Figure 2 compares precataract surgery VAadjusted survival functions by treatment group. 
Table I Patient characteristics and univariate tests of between-group differences

\begin{tabular}{|c|c|c|c|c|c|c|c|}
\hline \multirow{2}{*}{$\begin{array}{l}\text { Means (M) and standard } \\
\text { deviations (SD) }\end{array}$} & \multicolumn{2}{|c|}{ Delayed $(N=12)$} & \multicolumn{2}{|c|}{ Same-day $(\mathbf{N}=\mathbf{2 3})$} & \multirow[b]{2}{*}{ Test } & \multirow[b]{2}{*}{ Statistic } & \multirow[b]{2}{*}{$P$-value } \\
\hline & $\bar{M}$ & SD & $\bar{M}$ & SD & & & \\
\hline Age at Final Visit & 81.37 & 9.21 & 84.43 & 8.41 & MW & -1.53 & $0.126 \dagger$ \\
\hline logMAR VA OP pre-CS & 0.57 & 0.40 & 0.69 & 0.56 & MW & -0.33 & 0.739 \\
\hline logMAR VA non-OP pre-CS & 0.26 & 0.19 & 0.30 & 0.24 & MW & -0.21 & 0.832 \\
\hline FU post-vitrectomy (months) & 54.82 & 37.74 & 43.70 & 33.26 & IS-t & 0.90 & 0.376 \\
\hline Total elapsed time (months) & 56.18 & 38.32 & 43.70 & 33.26 & IS-t & 1.00 & 0.324 \\
\hline logMAR VA OP final & 0.65 & 0.74 & 0.30 & 0.47 & MW & 1.51 & $0.130 \dagger$ \\
\hline logMAR VA non-OP final & 0.21 & 0.18 & 0.28 & 0.33 & MW & -0.04 & 0.972 \\
\hline Visual Utility-better eye $\mathrm{e}^{\mathrm{a}}$ & 0.85 & 0.07 & 0.89 & 0.08 & IS-t & -1.28 & 0.210 \\
\hline Visual Utility-both eyes ${ }^{\mathrm{a}}$ & 0.91 & 0.07 & 0.94 & 0.05 & MW & -1.54 & $0.124 \dagger$ \\
\hline Diff MD glaucoma progression & -5.62 & 2.96 & 0.17 & 3.41 & IS-t & -2.88 & $0.016^{*}$ \\
\hline Diff PSD glaucoma progression & 2.00 & 2.76 & -1.20 & 1.11 & MW & 2.55 & $0.011 *$ \\
\hline Counts (n) and proportions & $\mathbf{n}$ & Prop & $\mathbf{n}$ & Prop & Test & & $P$-value \\
\hline \# right eye & 6 & $50.0 \%$ & 13 & $56.5 \%$ & Fischer exact & & 0.736 \\
\hline \# w/pseudo. pre-CS & 3 & $25.0 \%$ & 7 & $30.4 \%$ & Fischer exact & & 1.000 \\
\hline \# w/polar cataract pre-CS & I & $8.3 \%$ & 0 & $0.0 \%$ & Fischer exact & & 0.343 \\
\hline \# w/cortical lens pre-CS & I & $8.3 \%$ & 4 & $17.4 \%$ & Fischer exact & & 0.640 \\
\hline \# w/glaucoma pre-CS & 4 & $33.3 \%$ & II & $47.8 \%$ & Fischer exact & & 0.489 \\
\hline \multicolumn{8}{|l|}{ Cataract nuclear sclerosis (NS) } \\
\hline $\mathrm{NS}=4+$ & 0 & $0.0 \%$ & 3 & $13.0 \%$ & Kendall's tau & & 0.813 \\
\hline $\mathrm{NS}=3+$ & 6 & $50.0 \%$ & 9 & $39.1 \%$ & & & \\
\hline $\mathrm{NS}=2+$ & 5 & $41.7 \%$ & 8 & $34.8 \%$ & & & \\
\hline $\mathrm{NS}=\mathrm{I}+$ & 0 & $0.0 \%$ & 2 & $8.7 \%$ & & & \\
\hline None & I & $8.3 \%$ & 1 & $4.3 \%$ & & & \\
\hline \multicolumn{8}{|c|}{ Posterior subcapsular cataract (PSC) } \\
\hline $\mathrm{PSC}=3+$ & I & $8.3 \%$ & 2 & $8.7 \%$ & Kendall's tau & & 0.531 \\
\hline $\mathrm{PSC}=2+$ & 2 & $16.7 \%$ & 1 & $4.4 \%$ & & & \\
\hline PSC $=1+$ & 0 & $0.0 \%$ & 2 & $8.7 \%$ & & & \\
\hline None & 9 & $75.0 \%$ & 18 & $78.3 \%$ & & & \\
\hline \multicolumn{8}{|l|}{ Intraocular lens type } \\
\hline posterior chamber & 4 & $33.3 \%$ & 1 & $4.6 \%$ & Fischer exact & & $0.084 \dagger$ \\
\hline anterior chamber & 4 & $33.3 \%$ & 13 & $59.1 \%$ & & & \\
\hline posterior sulcus & 4 & $33.3 \%$ & 8 & $36.4 \%$ & & & \\
\hline \multicolumn{8}{|l|}{ Volume of retained lens fragments } \\
\hline Large & I & $11.1 \%$ & 19 & $100.0 \%$ & Kendall's tau & & $0.000 * * *$ \\
\hline Moderate & 5 & $55.6 \%$ & 0 & $0.0 \%$ & & & \\
\hline Small & 3 & $33.3 \%$ & 0 & $0.0 \%$ & & & \\
\hline missing data & 3 & & 4 & & & & \\
\hline \# new glaucoma cases & I & $12.5 \%$ & 2 & $15.4 \%$ & Fischer exact & & 1.000 \\
\hline \# w/final retinal condition & 6 & $50.0 \%$ & 1 & $4.3 \%$ & Fischer exact & & $0.003^{* *}$ \\
\hline \# w/retinal detachment & 2 & $16.7 \%$ & I & $4.3 \%$ & Fischer exact & & 0.266 \\
\hline \# w/cystoid macular edema & 3 & $25.0 \%$ & 3 & $13.0 \%$ & Fischer exact & & 0.391 \\
\hline \# w/final anterior chamber cells & 0 & $0.0 \%$ & 2 & $8.7 \%$ & Fischer exact & & 0.536 \\
\hline \# w/final corneal condition & 2 & $16.7 \%$ & 1 & $4.3 \%$ & Fischer exact & & 0.266 \\
\hline$\#$ w/VA Snellen final $<20 / 40$ & 5 & $41.7 \%$ & 5 & $21.7 \%$ & Fischer exact & & 0.258 \\
\hline$\#$ w/VA Snellen final $\leq 20 / 200$ & 3 & $25.0 \%$ & I & $4.3 \%$ & Fischer exact & & $0.106 \dagger$ \\
\hline
\end{tabular}

Notes: $* P \leq 0.05, * * P \leq 0.01, * * * P \leq 0.001,+P \leq 0.15$. All data are for the operated eye unless noted. ${ }^{2} V$ isual utility analyses included 22 same-day patients. Visual utilitybetter eye: $0.92=20 / 20$ with $<20 / 40$ in the other eye, $0.87=20 / 25,0.84=20 / 30 .{ }^{16}$ Visual utility-both eyes: $0.96=$ better eye 20/20-20/40 and worse eye $>20 / 200,0.88$ $=$ better eye 20/50-20/80 and worse eye $>20 / 200 .{ }^{17}$

Abbreviations: Diff, Differential; FU, follow up time in months; IS-t, independent-samples $t$-test; MD, mean deviation; MW, Mann-Whitney rank-sum test; N, n, number of eyes; non-OP, non-operated eye; OP, operated eye; pre-CS, before cataract surgery; prop, proportion; PSD, pattern specific deviation; pseudo, pseudoexfoliation; VA, visual acuity; w/, with. 

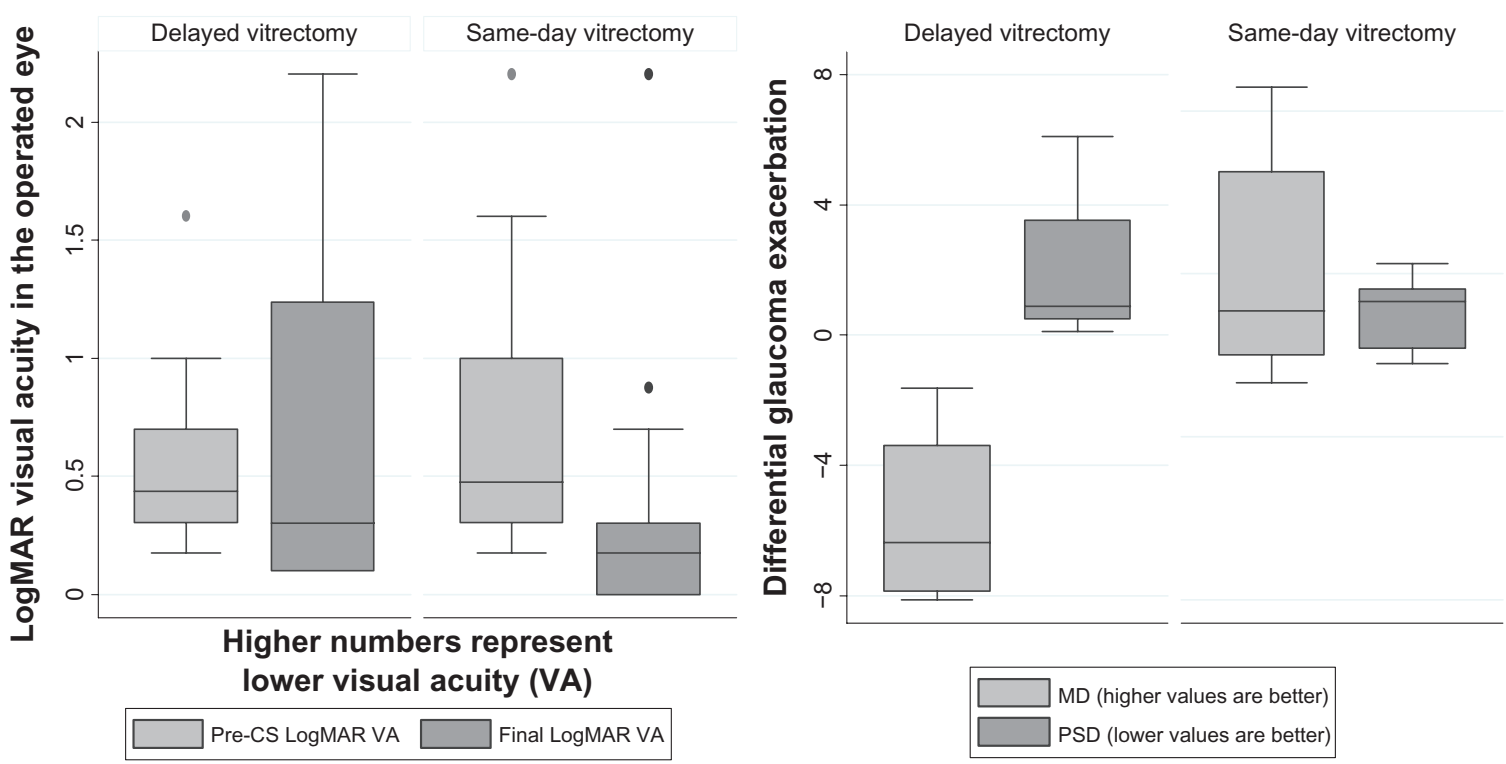

Figure I Box-and-whisker plot comparisons of precataract surgery (Pre-CS) logMAR visual acuity (VA) and final logMAR VA in the operated eye and differential glaucoma progression (exacerbation) (mean deviation [MD] and pattern-specific deviation [PSD]) by treatment group.

Data for differential glaucoma progression were available for only four delayed and eight same-day patients. Both the mean MD differential (difference $=-5.79,95 \% \mathrm{CI}=-10.27$, $-1.32, t=-2.88, P=0.016$ ) and the mean PSD differential (difference $=1.66, \mathrm{Z}=2.55, P=0.011)$ were significant $($ Table 5 , models 6 and 7) and indicated greater glaucoma-related loss of sensitivity in delayed vitrectomy eyes. Table 3 shows estimated mean final MD and PSD for the operated eye, based on precataract surgery values and actual changes in the fellow eye (data not shown) if the patients had been in the other group.

Table 2 Descriptive statistics and normal distribution tests

\begin{tabular}{|c|c|c|c|c|c|c|c|c|c|c|}
\hline \multirow[t]{2}{*}{ Variable and its function } & \multicolumn{3}{|c|}{ Delayed PPV $(n=12)$} & \multicolumn{3}{|c|}{ Same-day PPV $(n=23)$} & \multicolumn{2}{|c|}{ Skew/kurtosis ${ }^{\mathrm{b}}$ test } & \multicolumn{2}{|c|}{ Shapiro-Wilk ${ }^{\mathrm{b}}$ test } \\
\hline & Min & Md & Max & Min & Md & Max & $\operatorname{adj} \chi^{2}$ & $P$-value & $\mathbf{Z}$ & $P$-value \\
\hline \multicolumn{11}{|c|}{ Independent or confounding variables } \\
\hline Age at final visit & 58.13 & 81.99 & 97.03 & 62.79 & 85.65 & 99.45 & 8.63 & $0.013^{*}$ & 2.20 & $0.014 *$ \\
\hline VA logMAR OP pre-CS & 0.176 & 0.438 & 1.602 & 0.176 & 0.477 & 2.204 & 10.88 & $0.004 * *$ & 3.92 & $0.000 * * *$ \\
\hline VA Snellen OP pre-CS & $20 / 800$ & $20 / 55$ & $20 / 30$ & $20 / 3077$ & $20 / 60$ & $20 / 30$ & 4.07 & 0.130 & -1.64 & 0.950 \\
\hline VA logMAR non-OP pre-CS & 0.000 & 0.176 & 0.544 & 0.000 & 0.301 & 1.000 & 12.44 & $0.002 * *$ & 2.89 & $0.002 * *$ \\
\hline VA Snellen non-OP pre-CS & $20 / 70$ & $20 / 30$ & $20 / 20$ & $20 / 200$ & $20 / 40$ & $20 / 20$ & 0.95 & 0.623 & -1.55 & 0.939 \\
\hline FU post-PPV (months) & 5.93 & 53.28 & 113.37 & 3.07 & 36.57 & 123.50 & 2.83 & 0.243 & 1.86 & $0.031 *$ \\
\hline Total elapsed time (months) & 6.33 & 56.57 & 115.30 & 3.07 & 36.57 & 123.50 & 2.85 & 0.240 & 1.89 & $0.030 *$ \\
\hline VA logMAR non-OP final & 0.000 & 0.018 & 0.544 & 0.000 & 0.097 & 1.301 & 18.35 & $0.000 * * *$ & 4.19 & $0.000 * * *$ \\
\hline VA Snellen non-OP final & $20 / 70$ & $20 / 30$ & $20 / 20$ & $20 / 400$ & $20 / 25$ & $20 / 20$ & 2.77 & 0.250 & 0.32 & 0.374 \\
\hline \multicolumn{11}{|l|}{ Dependent variables } \\
\hline VA logMAR OP final & 0.097 & 0.301 & 2.204 & 0.000 & 0.176 & 2.204 & 18.23 & $0.000 * * *$ & 5.05 & $0.000 * * *$ \\
\hline VA Snellen OP final & $20 / 3200$ & $20 / 40$ & $20 / 25$ & $20 / 3200$ & $20 / 30$ & $20 / 20$ & 3.24 & 0.198 & -0.39 & 0.651 \\
\hline Visual utility - better eye $\mathrm{e}^{\mathrm{a}}$ & 0.74 & 0.84 & 0.97 & 0.77 & 0.87 & 1.00 & 4.15 & 0.126 & 1.00 & 0.160 \\
\hline Visual utility - both eyes ${ }^{\mathrm{a}}$ & 0.805 & 0.96 & 0.96 & 0.83 & 0.96 & 0.96 & 7.27 & $0.026^{*}$ & 3.04 & $0.001 * *$ \\
\hline Diff MD glaucoma prog & -8.12 & -6.37 & -1.63 & -3.35 & -1.15 & 5.72 & 0.21 & 0.899 & -0.63 & 0.737 \\
\hline Diff PSD glaucoma prog & 0.11 & 0.895 & 6.11 & -2.76 & -0.85 & 0.31 & 10.38 & $0.006 * *$ & 2.10 & $0.018^{*}$ \\
\hline
\end{tabular}

Notes: $* P \leq 0.05 ; * * P \leq 0.01 ; * * * P \leq 0.00 I ;{ }^{\dagger} P \leq 0.15$. ${ }^{a} V$ isual utility analyses included 22 same-day patients. Visual utility - better eye: I.00 $=20 / 20$ bilaterally, permanently; $0.97=20 / 20$ with $20 / 20$ to $20 / 25$ in the other eye, $0.92=20 / 20$ with $\leq 20 / 40$ in the other eye, $0.87=20 / 25,0.84=20 / 30,0.80=20 / 40,0.77=20 / 50,0.74=20 / 70 .{ }^{16}$ Visual utility - both eyes: 0.96 = better eye $20 / 20$ to $20 / 40$ and worse eye $>20 / 200,0.88$ = better eye $20 / 50$ to $20 / 80$ and worse eye $>20 / 200,0.83=$ better eye $20 / 20$ to $20 / 40$ and worse eye $<20 / 200,0.88$ = better eye $20 / 50$ to $20 / 80$ and worse eye $<20 / 200$; ${ }^{17}$ b the null hypothesis for the Skew/kurtosis test and the Shapiro-Wilk test is that the data come from a normal distribution.

Abbreviations: adj $\chi^{2}$, adjusted chi-square statistic; Diff, differential; FU, follow-up time in months; Max, maximum; Md, median; Min, minimum; n, number of eyes; non-OP, nonoperated eye; OP, operated eye; post-PPV, after pars plana vitrectomy; PPV, pars plana vitrectomy; pre-CS, before cataract surgery; prog, progression; VA, visual acuity; Z, Z statistic. 
Table 3 Summary comparison of outcomes and actual vs estimated (based on statistical models) if patients had been in the other treatment group

\begin{tabular}{|c|c|c|c|c|c|c|c|}
\hline \multirow[t]{2}{*}{ Variable and model } & \multirow[t]{2}{*}{ Treatment group } & \multirow[t]{2}{*}{$\mathbf{n}$} & \multicolumn{2}{|c|}{$\begin{array}{l}\text { Actual treatment } \\
\text { group means }\end{array}$} & \multicolumn{3}{|c|}{$\begin{array}{l}\text { Estimated means if patients had been in the } \\
\text { other treatment group }\end{array}$} \\
\hline & & & Pre-CS & Final & Unadjusted & Adjusted for age & $\begin{array}{l}\text { Adjusted for } \\
\text { pre-CS VA }\end{array}$ \\
\hline Final VA in the operated & Same-day PPV eyes & 23 & $20 / 98$ & $20 / 40$ & $20 / 90$ & $20 / 106$ & $20 / 112$ \\
\hline $\begin{array}{l}\text { eye (Table 4, model IA } \\
\text { and B) }\end{array}$ & Delayed PPV eyes & 12 & $20 / 75$ & $20 / 90$ & $20 / 40$ & $20 / 34$ & $20 / 32$ \\
\hline Visual utility - better & Same-day patients & 22 & $N / A$ & 0.89 & 0.85 & 0.89 & 0.89 \\
\hline $\begin{array}{l}\text { eye }^{\mathrm{a}} \text { (Table } 4 \text {, model } 2 \mathrm{~A} \\
\text { and } \mathrm{B} \text { ) }\end{array}$ & Delayed patients & 12 & N/A & 0.85 & 0.89 & 0.84 & 0.85 \\
\hline Mean deviation & Same-day PPV eyes & 8 & -11.07 & -10.32 & -17.33 & $\mathrm{~N} / \mathrm{A}$ & N/A \\
\hline (Table 5, model 6) & Delayed PPV eyes & 4 & -5.53 & -8.33 & -2.53 & $\mathrm{~N} / \mathrm{A}$ & $\mathrm{N} / \mathrm{A}$ \\
\hline Pattern-specific & Same-day PPV eyes & 8 & 6.58 & 5.95 & 9.65 & $N / A$ & $\mathrm{~N} / \mathrm{A}$ \\
\hline $\begin{array}{l}\text { deviation } \\
\text { (Table 5, model 7) }\end{array}$ & Delayed PPV eyes & 4 & 3.93 & 5.91 & 2.70 & $\mathrm{~N} / \mathrm{A}$ & $\mathrm{N} / \mathrm{A}$ \\
\hline
\end{tabular}

Notes: ${ }^{\text {V } V i s u a l ~ u t i l i t y ~ a n a l y s i s ~ i n c l u d e d ~} 22$ same-day patients. Visual utility - better eye: $0.92=20 / 20$ with $\leq 20 / 40$ in the other eye, $0.87=20 / 25,0.84=20 / 30 .{ }^{16}$ Abbreviations: age, age at final visit; n, number of eyes; N/A, not applicable to this analysis because the adjustments based on these variables were not significant; PPV, pars plana vitrectomy; pre-CS, before cataract surgery; VA, visual acuity.

\section{Discussion}

The reported incidence of RLF following cataract surgery varied between $0.1 \%$ and $1.6 \%,,^{2,7,23,24}$ sometimes higher for inexperienced surgeons. ${ }^{3,6,25}$ Our rate of $0.46 \%(35 / 7565)$ represents the 11-year history at MCF, a single academic institution, with all cataract surgeries performed by five experienced, fellowship-trained cataract surgeons. During this time, MCF developed its no-move, no-wait (more than 15 minutes) policy for same-day vitrectomies. If a cataract surgeon experiences an intraoperative complication with posteriorly dislocated lens fragments, he immediately calls the on-site vitreoretinal surgeon for assistance. The immediate surgical goals include complete pars plana removal of the lens fragments with placement of an IOL. All RLF cases during the past 6 years were handled in this manner.

Although some eyes with crystalline RLF are treated medically (at other facilities) with good long-term outcomes, most develop sufficiently severe postoperative complications (elevated IOP, visual obscuration, and inflammation) to warrant removal by vitrectomy. This may be performed during the same surgical episode (depending on the availability, experience, and philosophy of the vitreoretinal surgeon) or delayed. Patient displeasure is common following complicated cataract surgery, ${ }^{1,4}$ and tends not to improve if reduced VA persists. ${ }^{26}$ One author reported that “ “.. most [RLF] patients, who had expected a rapid visual recovery after cataract surgery, were very dissatisfied with poor vision post-operatively." 4 Same-day vitrectomy, when feasible, could mitigate patient dissatisfaction, ${ }^{27}$ prevent the need for a second procedure, and hasten visual recovery. ${ }^{28}$
Some previous studies have suggested that same-day vitrectomy yields better outcomes ${ }^{5,7,8,29}$ whereas others have reported poorer same-day outcomes., ${ }^{3,11,23,25}$ For good VA ( $\geq 20 / 40)$, three studies favored same-day vitrectomy by $>30 \%$ : Chen et $\mathrm{al}^{8}(36.2 \%)$, Romero-Aroca et $\mathrm{al}^{7}$ (35.7\%), and Stefaniotou et $\mathrm{al}^{5}(31.9 \%)$; while three others favored delayed vitrectomy by $>10 \%$ : Stilma et $\mathrm{al}^{23}(26.3 \%)$, Borne et $\mathrm{al}^{25}$ (18.75), and Tajunisah and Reddy ${ }^{3}$ (12.5\%). For bad VA $(\leq /<20 / 200)$, two studies favored same-day vitrectomy by $>20 \%$ : Stefaniotou et $\mathrm{al}^{5}(27.8 \%)$ and Chen et $\mathrm{al}^{8}(22.0 \%)$; while two others favored delayed vitrectomy by $\geq 20 \%$ : Tajunisah and Reddy ${ }^{3}(42.9 \%)$ and Watts et $a^{11}$ (20.0\%). Compared to our delayed group, our same-day group included more patients with good final VA (78.3\% $[18 / 23]$ versus $58.3 \%$ [7/12], difference $=20.7 \%$ ); fewer patients with bad final VA $(4.3 \%$ [1/23] versus $25.0 \%$ [3/12], difference $=20.7 \%$ ); and better mean (unadjusted) final VA (20/40 versus $20 / 90$ ), despite having poorer mean initial VA (20/98 versus 20/75), and older mean age ( $3+$ years).

None of these previous studies randomized patients to same-day or delayed vitrectomy, so perhaps these inconsistent same-day versus delayed results have been confounded by different practice patterns and noncomparable treatment groups. Some inconsistency might be due to the fact that many previous studies included same-day vitrectomy patients who were transported between facilities and had much longer ( $>15$ minutes) wait between cataract surgery and vitrectomy. No stratification (by waiting time or location of cataract surgery) was found in any previous analysis of same-day versus delayed vitrectomy outcomes. If waiting 


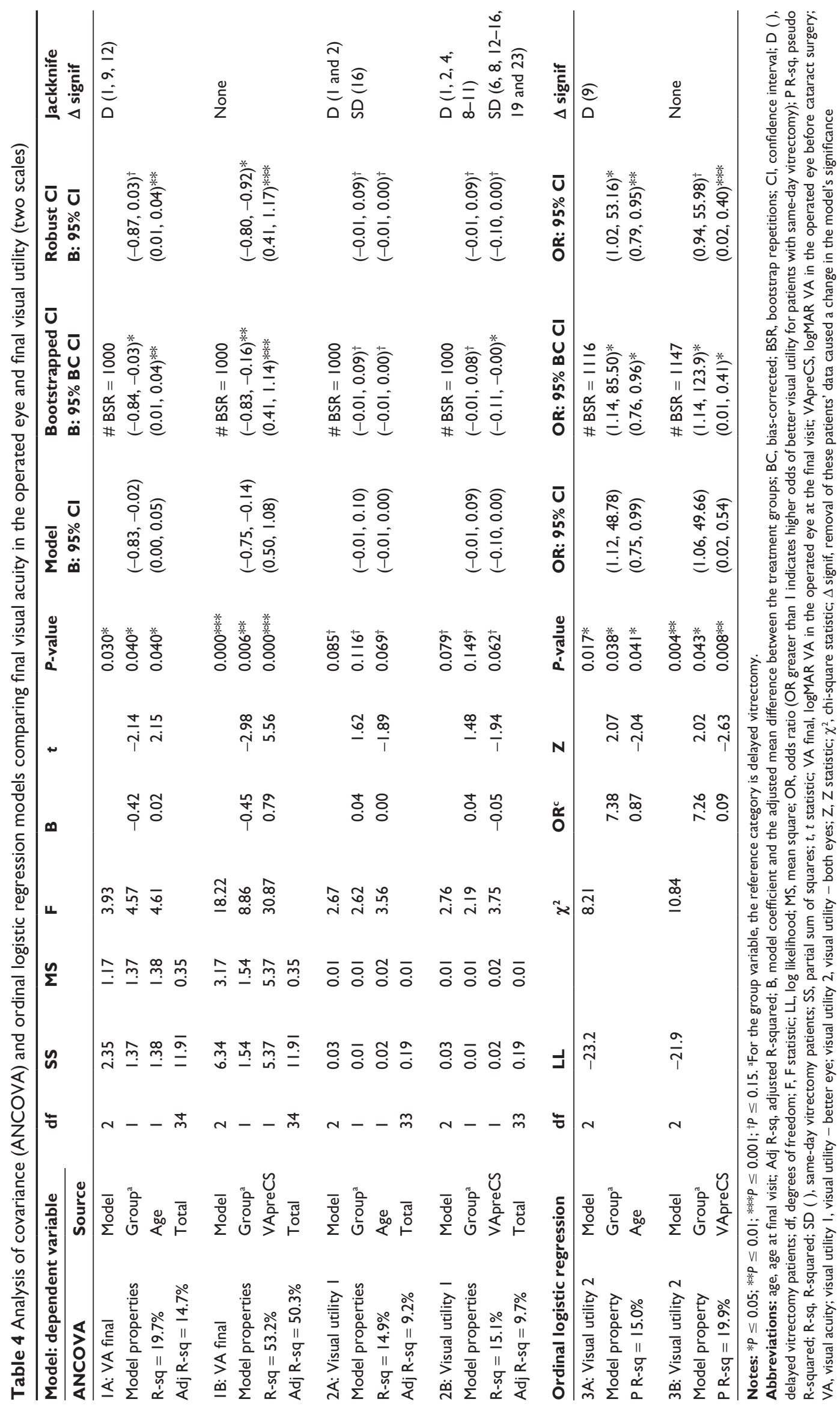




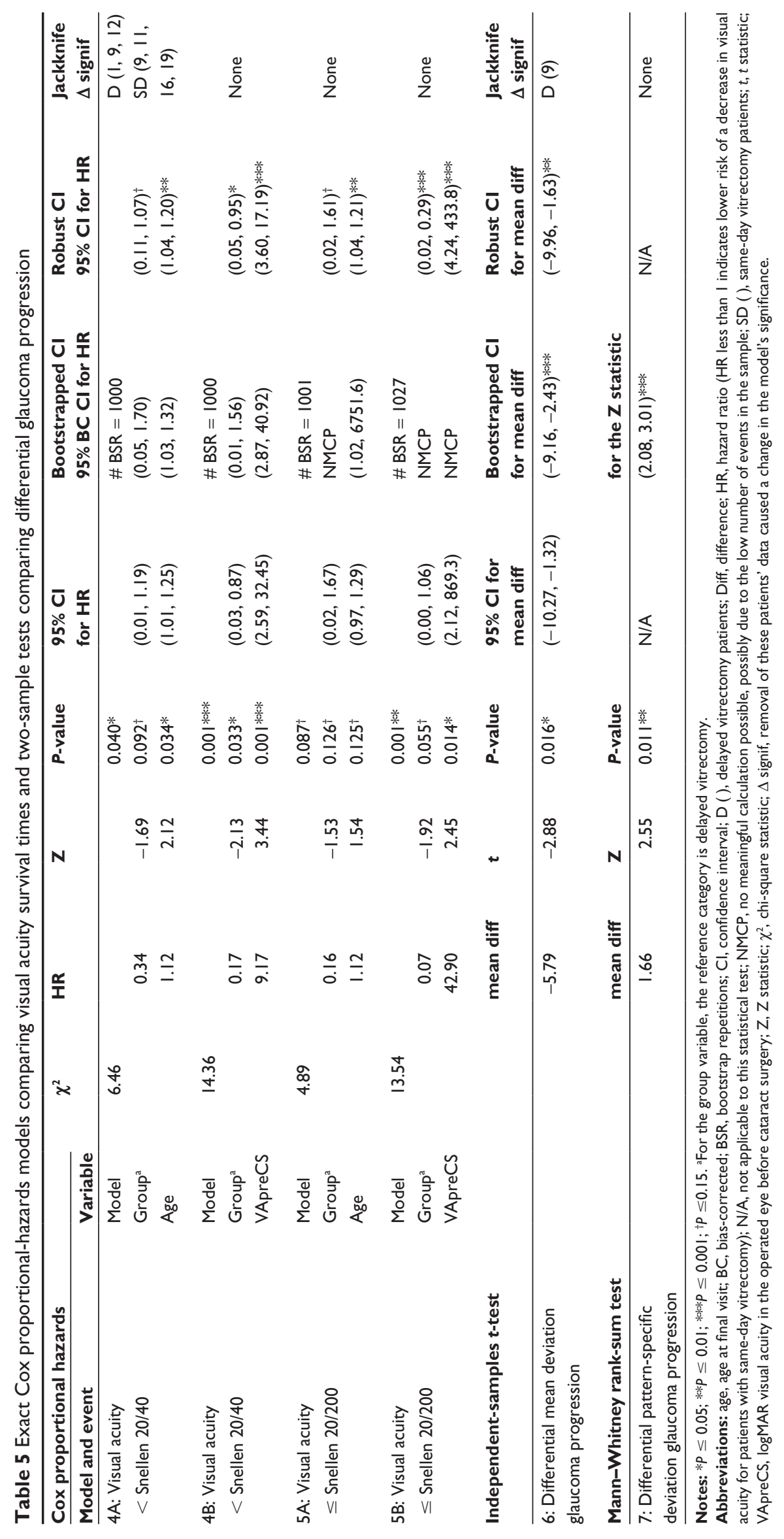



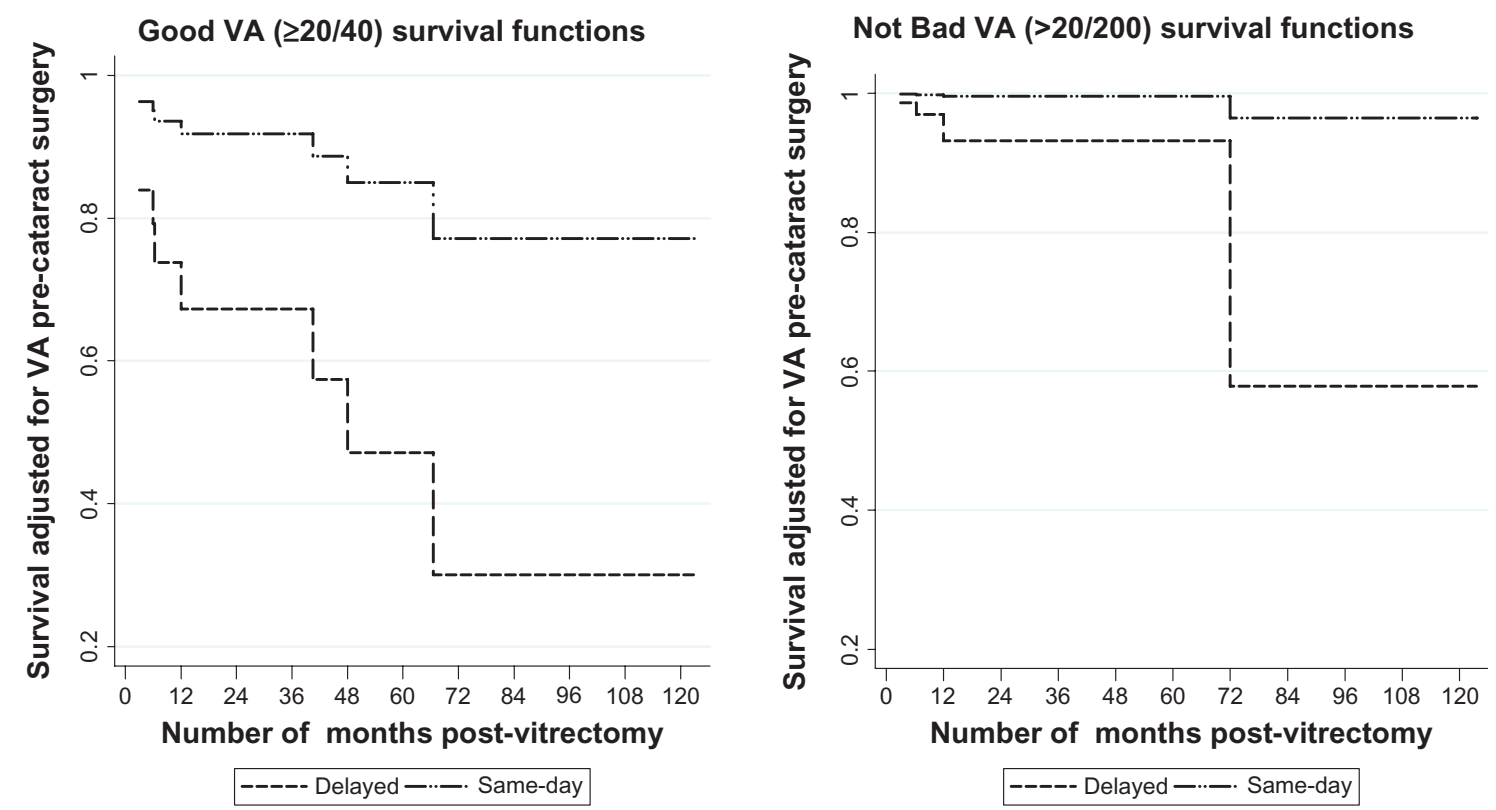

Figure 2 Comparison of Cox proportional-hazards survival functions for good visual acuity (VA $\geq 20 / 40$ ) and not bad visual acuity (VA $>20 / 200)$, in the operated eye, by treatment group, adjusted for precataract surgery VA in the operated eye.

time and/or patient movement between surgeries are important factors in the relative success of same-day vitrectomy, then policy differences among facilities could explain why, in some (but not all) studies, same-day vitrectomy produced better outcomes.

Perhaps immediate vitrectomy (within 15 minutes) prevents time-dependent inflammation and the accompanying choroidal congestion that occurs with same-day patient transfer between different surgical facilities. Immediate vitrectomy for RLF closely resembles a planned pars plana lensectomy, something frequently performed without complications by vitreoretinal surgeons. Compared to waiting several hours between surgeries, immediate vitrectomy may take advantage of a clear cornea and minimally inflamed eye to enable better removal of RLF with fewer complications.

RLF have been linked to new cases of glaucoma, ${ }^{28,30}$ and although we did not observe a significant difference in new glaucoma cases, we discovered that vitrectomy timing may affect the progression of preexisting glaucoma. Our same-day eyes with preexisting glaucoma experienced less differential visual field loss (glaucoma progression, measured by both MD and PSD, using fellow eyes as controls). Although possible reasons that same-day vitrectomy might have a stabilizing effect on glaucoma are not known, perhaps same-day vitrectomy prevents time-dependent damage to the trabecular meshwork. ${ }^{30}$ Eyes with RLF begin to develop macrophage-mediated inflammation after 3 days, ${ }^{29,31}$ and these large cells may exacerbate preexisting glaucoma by further affecting the already-compromised aqueous outflow. ${ }^{29}$ No other analyses of the effect of vitrectomy timing on the progression of preexisting glaucoma were found in the literature, nor were other analyses found of visual utility and RLF removal timing.

\section{Study limitations}

The primary study limitation was the small, nonrandomized sample. A description of our attempts to minimize its effect may be found in the appendix. Our patients were not randomized to vitrectomy timing, because our preferred practice pattern evolved from mostly delayed vitrectomies in the early study period to exclusively same-day vitrectomies in the latter study years. However, there were no significant between-group differences in any precataract surgery variables. We used several statistical techniques, including robust CIs, bootstrapping, and jackknifing to increase confidence in our small sample's results. In general, robust CIs and bootstrapping validated the primary models' results (Tables 4 and 5).

\section{Future research}

Future researchers, who may have more data, can attempt to verify these findings. We suggest future analyses comparing outcomes for same-day versus delayed vitrectomy stratified by cataract surgery location and adjusting for potential confounding variables (eg, age and precataract surgery VA). Statistical adjustment can mitigate the selection bias 
inherent in nonrandomized studies, and stratification should yield more homogeneous treatment groups and help determine if vitrectomy delay and/or patient transport between facilities had an effect on the relative success of same-day vitrectomy. Outcomes should be compared for same-day and delayed patients, all of whom had cataract surgery at the same facility as the vitrectomy, possibly with subanalyses limiting the same-day group to only those patients who were not moved to another operating room and who did not wait more than 15 minutes (or another specified time) between cataract surgery and vitrectomy. Separately, outcomes should be compared for same-day and delayed patients, none of whom had cataract surgery at the same facility as the vitrectomy.

\section{Conclusion}

These results suggest that same-day vitrectomy for RLF at MCF (performed based on our no-move, no-wait policy) is associated with superior long-term outcomes. Readers should not generalize these results to RLF patients whose same-day vitrectomy was done under different policies for patient waiting and transport. Furthermore, eyes with preexisting glaucoma may benefit from same-day vitrectomy, a result not previously found in the literature. Though our practice's ability to immediately summon a vitreoretinal surgeon to the operating room is not unique, this is not reproducible for many cataract surgeons. Because the previously published data on same-day vitrectomy are mixed, surgeons should carefully examine their own results to determine if this strategy is appropriate for their practices.

\section{Acknowledgments}

The authors would like to acknowledge Professors John Rizzo, Debra S Dwyer, and A Laurie Shroyer for their review and approval of the data analysis plan.

\section{Disclosure}

The authors report no conflicts of interest in this work.

\section{References}

1. Stewart MW. Managing retained lens fragments: raising the bar. Am J Ophthalmol. 2009;147(4):569-570.

2. Stenkula S, Byhr E, Crafoord S, et al. Tackling the "dropped nucleus". Acta Ophthalmol Scand. 1998;76(2):220-223.

3. Tajunisah I, Reddy SC. Dropped nucleus following phacoemulsification cataract surgery. Med J Malaysia. 2007;62(5):364-367.

4. Tommila P, Immonen I. Dislocated nuclear fragments after cataract surgery. Eye (Lond). 1995;9(Pt 4):437-441.

5. Stefaniotou M, Aspiotis M, Pappa C, Eftaxias V, Psilas K. Timing of dislocated nuclear fragment management after cataract surgery. $J$ Cataract Refract Surg. 2003;29(10):1985-1988.
6. Hutton WL, Snyder WB, Vaiser A. Management of surgically dislocated intravitreal lens fragments by pars plana vitrectomy. Ophthalmology. 1978;85(2):176-189.

7. Romero-Aroca P, Fernandez-Ballart J, Mendez-Marin I, Salvat-Serra M, Baget-Bernaldiz M, Buil-Calvo JA. Management of nucleus loss into the vitreous: long term follow up in 63 patients. Clin Ophthalmol. 2007;1(4):505-512.

8. Chen CL, Wang TY, Cheng JH, Tai MC, Lu DW, Chen JT. Immediate pars plana vitrectomy improves outcome in retained intravitreal lens fragments after phacoemulsification. Ophthalmologica. 2008;222(4):277-283.

9. Kim JE, Flynn HW Jr, Smiddy WE, et al. Retained lens fragments after phacoemulsification. Ophthalmology. 1994;101(11):1827-1832.

10. Gonvers M. New approach to managing vitreous loss and dislocated lens fragments during phacoemulsification. J Cataract Refract Surg. 1994;20(3):346-349.

11. Watts P, Hunter J, Bunce C. Vitrectomy and lensectomy in the management of posterior dislocation of lens fragments. $J$ Cataract Refract Surg. 2000;26(6):832-837.

12. Kageyama T, Ayaki M, Ogasawara M, Asahiro C, Yaguchi S. Results of vitrectomy performed at the time of phacoemulsification complicated by intravitreal lens fragments. Br J Ophthalmol. 2001;85(9):1038-1040.

13. Scott IU, Flynn HW Jr, Smiddy WE, et al. Clinical features and outcomes of pars plana vitrectomy in patients with retained lens fragments. Ophthalmology. 2003;110(8):1567-1572.

14. Bland M. An Introduction to Medical Statistics. 3rd ed. Oxford: Oxford University Press; 2000.

15. Moseley MJ, Jones HS. Visual acuity: calculating appropriate averages. Acta Ophthalmol. 1993;71:296-300.

16. Brown GC, Brown MM, Brown HC, Kindermann S, Sharma S. The goal of value-based medicine analyses: comparability. The case for neovascular macular degeneration. Trans Am Ophthalmol Soc. 2007;105:160-171.

17. Szabo SM, Beusterien KM, Pleil AM, et al. Patient preferences for diabetic retinopathy health states. Invest Ophthalmol Vis Sci. 2010;51(7):3387-3394.

18. Agresti A. An Introduction to Categorical Data Analysis. 2nd ed. Hoboken: John Wiley \& Sons; 2007.

19. Vittinghoff E, Glidden DV, Shiboski SC, McCulloch CE. Regression Methods in Biostatistics: Linear, Logistic, Survival, and Repeated Measures Models. New York: Springer; 2005.

20. Iezzoni LI. Risk Adjustment for Measuring Health Care Outcomes. 3rd ed. Chicago: Health Administration Press; 2003.

21. Pagano M, Gauvreau K. Principles of Biostatistics. 2nd ed. Pacific Grove: Duxbury; 2000.

22. Daniel WW. Biostatistics: A Foundation for Analysis in the Health Sciences. 5th ed. New York: John Wiley \& Sons; 1991.

23. Stilma JS, van der Sluijs FA, van Meurs JC, Mertens DA. Occurrence of retained lens fragments after phacoemulsification in The Netherlands. J Cataract Refract Surg. 1997;23(8):1177-1182.

24. Margherio RR, Margherio AR, Pendergast SD, et al. Vitrectomy for retained lens fragments after phacoemulsification. Ophthalmology. 1997;104(9):1426-1432.

25. Borne MJ, Tasman W, Regillo C, Malecha M, Sarin L. Outcomes of vitrectomy for retained lens fragments. Ophthalmology. 1996;103(6): 971-976.

26. Lu H, Jiang YR, Grabow HB. Managing a dropped nucleus during the phacoemulsification learning curve. J Cataract Refract Surg. 1999;25(3):447-450.

27. Dickinson PJ, Greven CM. Avoiding and managing the dislocated crystalline lens. Compr Ophthalmol Update. 2006;7(3):113-127.

28. Kwok AK, Li KK, Lai TY, Lam DS. Pars plana vitrectomy in the management of retained intravitreal lens fragments after cataract surgery. Clin Experiment Ophthalmol. 2002;30(6):399-403.

29. Yeo LM, Charteris DG, Bunce C, Luthert PJ, Gregor ZJ. Retained intravitreal lens fragments after phacoemulsification: a clinicopathological correlation. Br J Ophthalmol. 1999;83(10):1135-1138. 
30. Murat Uyar O, Kapran Z, Akkan F, Cilsim S, Eltutar K. Vitreoretinal surgery for retained lens fragments after phacoemulsification. Eur J Ophthalmol. 2003;13(1):69-73.

31. Wilkinson CP, Green WR. Vitrectomy for retained lens material after cataract extraction: the relationship between histopathologic findings and the time of vitreous surgery. Ophthalmology. 2001;108(9): 1633-1637.

32. Vanner EA, Stewart MW. Vitrectomy timing for retained lens fragments after surgery for age-related cataracts: a systematic review and meta-analysis. Am J Ophthalmol. 2011;152(3):345-351.
33. Remler D, Van Ryzin G. Research Methods in Practice: Strategies for Description and Causation. Los Angeles: Sage; 2011.

34. Bailey D. Research for the Health Professional. 2nd ed. Philadelphia: FA Davis; 1997.

35. Kane R. Understanding Health Care Outcomes Research. 2nd ed. Sudbury: Jones and Bartlett; 2006.

36. Shi L. Health Services Research Methods. 2nd ed. Clifton Park: Delmar Cengage Learning; 2008. 


\section{Appendix: how we enhanced the analysis of our small, nonrandomized sample}

\section{Statistical techniques and interpretation}

Bootstrapping and robust confidence intervals

The robustness of our models' results was assessed by bootstrapping and estimating robust CIs (Tables 4 and 5). Bootstrapping involves repeated sampling (with replacement) of patients from the same treatment group in the original data set. In the bootstrapped samples (at least 1000 samples for each analysis), each treatment group (same-day and delayed) had the same number of eyes as in the original data set. The same model was built with the bootstrapped samples as had been built with the original sample, and CIs were estimated based on the middle 95\% of these models' results. ${ }^{19}$ In general, the bootstrapped and robust CIs supported the same conclusions as did the primary models. Unstable bootstrapping results for the Cox proportional-hazards regressions (no meaningful calculations were possible) are possibly due to the small number of events in the sample, because the robust CI results did agree with the primary models.

\section{Jackknifing}

Jackknifing assessed our models' sensitivity to the exclusion of each eye's data, by rebuilding the models, deleting one observation at a time. ${ }^{19}$ Sensitivity to a single observation is common with small data sets, and should not be interpreted as invalidating the original analysis, but rather emphasizes that the results need to be confirmed with larger data sets.

The jackknifing analyses indicated that, while six models were not sensitive to the exclusion of any eye's data, six models were sensitive, although often a model's results remained marginally significant when one eye's data were removed. Patients whose data removal changed the significance of two or more models included four delayed vitrectomy patients and two same-day vitrectomy patients. One of these delayed vitrectomy patients had the longest cataract surgery to vitrectomy delay. If delayed vitrectomy does yield inferior results, ${ }^{32}$ it is possible that the patient with the longest delay would show a greater effect. The other three delayed vitrectomy patients were the oldest and had the worst precataract surgery and final VA. Perhaps the effects of a delayed vitrectomy are more acute for older patients and/or those with worse precataract surgery VA. On the other hand, the two same-day vitrectomy patients whose data changed the significance of two or more models were among the youngest, with the best precataract surgery and final VA, so perhaps any benefits of a same-day vitrectomy are greater for younger patients and/ or those with better precataract surgery VA.

\section{Statistical test interpretation}

In the literature, many analyses that do not achieve statistical significance are reported as indicating no effect: a common, but not entirely accurate interpretation of the results. Any study with a difference between groups indicates an effect. There is nothing magical about alpha, the significance level of a statistical test. At the "stroke" of 0.05 , the treatment effect does not turn into a pumpkin. The effect goes from significant to nonsignificant, but it does not vanish, like Cinderella's coach. Interpretation of statistical test results involves assessing whether or not this difference is clinically significant and sufficiently likely to be generalizable to the population from which the sample was drawn. This is why it is appropriate to note marginally significant results, especially when analyzing small samples, which tend to have low power to detect significant differences. ${ }^{14}$

\section{Appropriate sample selection}

In any treatment comparison, confidence in the results depends on the size, representativeness, and homogeneity of the sample and the balance of patient characteristics between treatment groups. Sample size and treatment group balance receive a great deal of attention, but representativeness and homogeneity of the sample receive little. However, homogeneity of the sample affects the estimated standard deviation used in power and sample size calculations, ${ }^{14}$ and representativeness of the sample is the foundation of external validity (below). ${ }^{33}$

Homogeneity of the sample refers to how similar the study's patients are, collectively - not across treatment groups. It is better, when comparing treatments, to have a smaller but relatively more homogeneous sample rather than a slightly larger but significantly more heterogeneous sample. When considering the appropriateness of a study with a nonrandomized comparison group, the " ... crucial question to ask is whether the two treatment groups truly come from the same population." ${ }^{34}$ Of particular importance in this (and any nonrandomized) study is that if you have a relatively homogeneous sample, you are more likely to have balanced treatment groups. Creating balanced treatment groups is, of course, the objective of random assignment (to treatment groups) in clinical trials.

When doing a retrospective study, researchers often have to make a trade-off between sample size and sample 
homogeneity. This study's delayed vitrectomy group included only patients who received cataract surgery at MCF, and who, therefore, would have been eligible for a same-day vitrectomy under the MCF no-move, no-wait policy. This inclusion criterion increased our sample's homogeneity and yielded the best nonrandomized delayed vitrectomy comparison group possible. ${ }^{34}$ During the period of this study, seven additional patients who did not receive their initial cataract surgery at MCF received a delayed vitrectomy for RLF at MCF, but were not included in this study. Had they been included, they would have confounded the analyses by significantly increasing the heterogeneity of the sample, because these patients were from a different population than that of the patients who received their initial cataract surgery at MCF.

In general, patients who are treated at different facilities are different in clinically significant ways, ${ }^{20}$ so nonrandomized studies that combine patients who where treated at different facilities will have a greater risk of unbalanced treatment groups, significant selection bias, and sample heterogeneity. This is especially true if, as in this case, the facility (where the patient received cataract surgery) is correlated with the treatment (no non-MCF cataract surgery patients received a same-day vitrectomy). For example, perhaps the patients who had cataract surgery at MCF were more likely to have been referred by other MCF doctors, and so could have been older and sicker than the typical cataract surgery patient. In fact, these seven additional (nonMCF cataract surgery) patients were significantly younger than the MCF cataract surgery patients, and their inclusion would have resulted in many significant baseline differences between the treatment groups. To summarize, in the absence of randomization, the best way to increase treatment group balance (through increased sample homogeneity) may be limiting the sample to patients of a single facility. ${ }^{35} \mathrm{By}$ only including patients who had cataract surgery at MCF, we limited the sample to a single population to minimize treatment group imbalances. ${ }^{34}$

\section{Assessing treatment group balance at baseline}

It is important to note that properly executed random assignment of patients to treatment groups, contrary to common belief, is unable to guarantee treatment group balance, especially for small samples. ${ }^{36}$ Instead, randomization only ensures that any imbalances are due simply to chance. ${ }^{14}$ In a randomized study, after randomization, one should use statistical tests to assess whether or not the treatment groups are in fact balanced on important variables. ${ }^{34}$ If no significant differences are found, in general, researchers would conclude that the randomization procedure created balanced treatment groups.

"To the extent that subjects from both experimental and control groups can be demonstrated to be similar in all important characteristics except for the intervention, the validity of the findings will be enhanced." 36 In this study, all univariate statistical tests for between-group differences at baseline (precataract surgery) were nonsignificant (none were even marginally significant; Table 1), including mean $\log$ MAR VA in the operated eyes (same-day $=0.69$, delayed $=0.57 ; \mathrm{Z}=-0.33, P=0.739)$ and nonoperated eyes (same-day $=0.30$, delayed $=0.26 ; \mathrm{Z}=0.21, P=0.832$ ). Therefore, if this had been a randomized study, and if the assessment of that randomization had produced the results in Table 1, these researchers would have concluded that the randomization procedure had created balanced treatment groups. This does not, in any way, imply that this study's treatment groups meet the criteria of randomization (that any imbalances are due only to chance) which is impossible to establish in a nonrandomized study. However, Table 1 indicates that there was no baseline between-group differences on any variable tested. This is the best possible statistical indication of balanced groups in a nonrandomized study, and is often not achieved, even in a randomized study.

\section{Assessing treatment group balance during the study}

Table 1 does indicate that the same-day and delayed vitrectomy groups were significantly different after cataract surgery on the variable for the volume of RLF. For patients with this data, those in the same-day group always had a large amount of lens fragments retained, which would tend to make the situation and prognosis worse, compared to patients with a lesser amount of RLF. For example, retinal detachment has been associated with a greater percentage of lens retained. ${ }^{24}$ Therefore, the fact that some delayed vitrectomy patients had only a small or moderate amount of lens fragments retained would tend to yield better outcomes in the delayed vitrectomy group. Since the delayed vitrectomy group did not have better outcomes, the treatment-group imbalance for the volume of RLF should not cause concern with the study's results. That is because this imbalance would tend to operate in the opposite direction, ie, causing the delayed group to have better outcomes, when in fact our study indicated that better outcomes were associated with same-day vitrectomy. 
Also, when studying the effects of RLF, ophthalmologists often have the luxury of using the nonoperated (fellow) eye (without the RLF) as a control. Table 1 indicates that there was no significant between-group difference in final logMAR $\mathrm{VA}$ in the fellow eye (same-day $=0.28$, delayed $=0.21$; $\mathrm{Z}=-0.04, P=0.972$ ). This does not prove, but lends credibility to, an assumption that no systemic issues (during the study period) adversely affected VA in one treatment group more than the other, and that therefore, the statistically significant group differences in adjusted final VA in the operated eye may be due to the continued presence of RLF in the delayed vitrectomy eyes.

\section{Other internal and external validity considerations}

\section{Internal validity}

When there is a small sample but many potential dependent variables, if a lot of analyses are performed, and the results collectively indicate that the same group (treatment) is better (as was the case in our study), each result lends support to the others. This does not obviate the issues of using a small, nonrandomized sample, but it can increase confidence in the results, albeit at a risk of increasing the probability of a type I error. ${ }^{14}$

Nonrandomized treatment comparisons can be valuable because “. ... such studies' outcomes were not defined or assessed for research purposes, but were conditions with objective, standard assessments, recorded before the study in a patient's record, as part of contemporary ophthalmologic practice. This reduces information and observer biases and patient misclassification, which sometimes occur during research studies." 32,36 Also, although data collection could not be masked, “ . .. all risk factors were recorded before the outcomes occurred in patients' medical records, which lack research biases." ${ }^{32,36}$

"For a natural or quasi experiment to be able to reveal genuine causal effects - for it to have good internal validity - two basic conditions are needed. First, the treatment or independent variable of interest must be exogenous. In other words, variation in the independent variable can't be driven by anything related to the outcome." 33 The MCF practice pattern evolved over time from mostly delayed vitrectomies to exclusively same-day vitrectomies. No clinical or demographic variable, in any way, influenced treatment group assignment. Therefore, in this study, treatment timing was indeed exogenous; that is, it was not based on anything that was related to any of the outcomes.

"Second, the treatment and comparison groups must be truly comparable - or homogeneous - the same in all relevant ways. For measured characteristics, the researcher can simply look at the available data to see how equivalent the treatment and comparison groups appear to be." ${ }^{33}$ As stated above, in this study, all univariate statistical tests for between-group differences at baseline (precataract surgery) were nonsignificant (Table 1). "For unmeasured characteristics, we cannot tell so easily and so must try to reason or guess if important unseen differences might lie beneath the surface." 33 As stated above, we increased the sample's homogeneity by including only patients who received cataract surgery at MCF. We are confident that this yielded the best comparison groups possible by minimizing unmeasured group differences. ${ }^{34}$

\section{External validity}

"The generalizability - or external validity - of quasi experiments and natural experiments often turns out to be better than in randomized field experiments." ${ }^{33}$ We avoided volunteer bias, which is present in prospective studies, because this sample was a consecutive series that included every RLF case from cataract surgery at MCF during the study years (1999-2010). ${ }^{36}$ Therefore, the results are generalizable, certainly to future patients at $\mathrm{MCF}{ }^{36}$ Additional studies are needed to determine if same-day vitrectomies, performed under a no-move, no-wait policy, might be associated with similar, superior long-term outcomes at other facilities.
Clinical Ophthalmology

\section{Publish your work in this journal}

Clinical Ophthalmology is an international, peer-reviewed journal covering all subspecialties within ophthalmology. Key topics include: Optometry; Visual science; Pharmacology and drug therapy in eye diseases; Basic Sciences; Primary and Secondary eye care; Patient Safety and Quality of Care Improvements. This journal is indexed on Submit your manuscript here: http://www.dovepress.com/clinical-ophthalmology-journal

\section{Dovepress}

PubMed Central and CAS, and is the official journal of The Society of Clinical Ophthalmology (SCO). The manuscript management system is completely online and includes a very quick and fair peer-review system, which is all easy to use. Visit http://www.dovepress.com/ testimonials.php to read real quotes from published authors. 